\title{
A theoretical study on the assembly and stabilization of a magic cluster
}

$\mathrm{Al}_{4} \mathbf{N}^{-}$

\section{Supporting information}

Li-ming Yang, Yi-hong Ding*, Chia-chung Sun

State Key Laboratory of Theoretical and Computational Chemistry, Institute of Theoretical Chemistry, Jilin University, Changchun 130023, People's Republic of China

(E-mail: yhdd@mail.jlu.edu.cn; Fax:+86-431-8498026)

\section{Full citations for ref 13e:}

13.(e) Gaussian03 (RevisionA.1), M. J. Frisch, G. W. Trucks, H. B. Schlegel, G. E. Scuseria, M. A. Robb, J. R. Cheeseman, J. A. Montgomery, Jr., T. Vreven, K. N. Kudin, J. C. Burant, J. M. Millam, S. S. Iyengar, J. Tomasi, V. Barone, B. Mennucci, M. Cossi, G. Scalmani, N. Rega, G. A. Petersson, H. Nakatsuji, M. Hada, M. Ehara, K. Toyota, R. Fukuda, J. Hasegawa, M. Ishida, T. Nakajima, Y. Honda,O. Kitao, H. Nakai, M. Klene, X. Li, J. E. Knox, H. P. Hratchian, J. B. Cross, C. Adamo, J. Jaramillo, R. Gomperts, R. E. Stratmann, O. Yazyev, A. J. Austin, R. Cammi, C. Pomelli, J. W. Ochterski, P. Y. Ayala, K. Morokuma, G. A. Voth, P. Salvador, J. J. Dannenberg, V. G. Zakrzewski, S. Dapprich, A. D. Daniels, M. C. Strain, O. Farkas, D. K. Malick, A. D. Rabuck, K. Raghavachari, J. B. Foresman, J. V. Ortiz, Q. Cui, A. G. Baboul, S. Clifford, J. Cioslowski, B. B. Stefanov, G. Liu, A. Liashenko, P. Piskorz, I. Komaromi, R. L. Martin, D. J. Fox, T. Keith, M. A. Al-Laham, C. Y. Peng, A. Nanayakkara, M. Challacombe, P. M.W. Gill, B. Johnson, W. Chen, M.W. Wong, C. Gonzalez, J. A. Pople, Gaussian, Inc., Pittsburgh, PA, 2003. 
Note: an imaginary frequency indicates the existence of a vibrational mode that is dynamically unstable and leads to a more stable structure. Transition states of a chemical reaction are saddle points exhibiting only one imaginary frequency. Saddle points with more dynamical systems with sufficiently high vibrational energy but are generally not of chemical significance.

In this report, NIMAG means the number of imaginary frequency of saddle point.

${ }^{\mathbf{m}} \mathbf{N}^{\mathbf{q}}$ : “ $\mathbf{m}$ ” means spin electron state (singlet, triplet), “ $\mathbf{N}$ ” means the energy order of various isomers, superscript “q-” means the charge of the total system.

\section{The calculated properties of $\mathbf{N}_{2} \mathrm{Al}_{8}{ }^{2-}$}

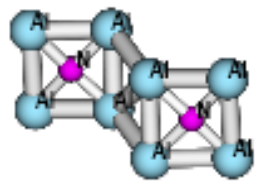

$\mathbf{1}_{1}(\mathrm{~s}-\mathrm{s})^{2-}$

0.00

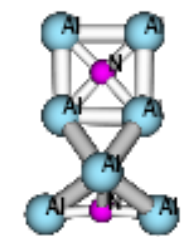

${ }^{1} 1(f-s-90)^{2-}$

(NIMAG=1)

$-2.80$

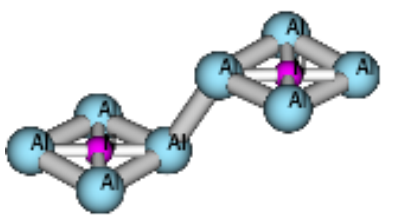

${ }^{1} \mathbf{1}(c-c)^{2-}$

1.71

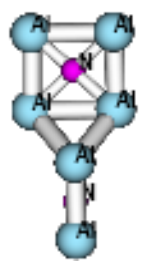

${ }^{1} 1(\mathrm{~s}-\mathrm{s}-90)^{2-}$

(NIMAG=1)

0.87

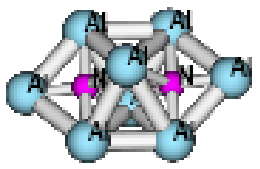

$-12.46$

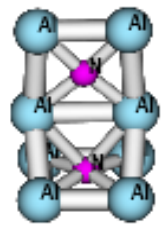

${ }^{1} 1(\mathrm{f}-\mathrm{s}-0)^{2-}$

(NIMAG=3)

20.44

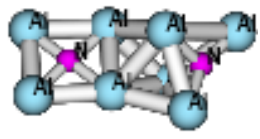

$-4.98$

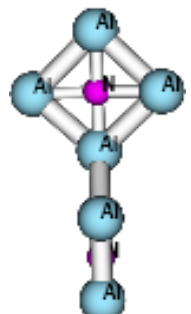

${ }^{1} 1(\mathrm{~s}-\mathrm{C}-90)^{2-}$

(NIMAG=3)

33.07

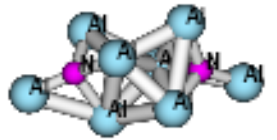

$-1.13$

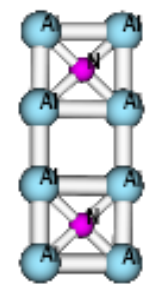

${ }_{1}^{1}(\mathrm{~s}-\mathrm{s}-0)^{2-}$

(NIMAG=4)

38.06

${ }^{1}\left(\mathrm{Al}_{4} \mathrm{~N}^{-}\right)_{2}$ 


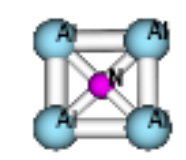

bi

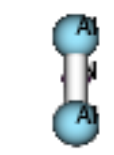

${ }^{1}{ }_{1(\mathrm{~s}-\mathrm{s}-90)^{-}}{ }^{1}{ }^{1} \mathbf{1}(\mathrm{s}-\mathrm{s}-0)^{-}$

0.00

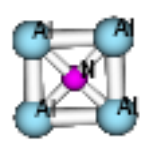

di

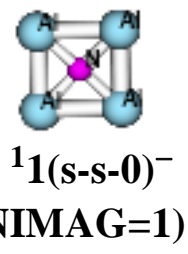

1.73

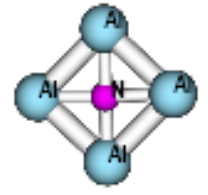

由)

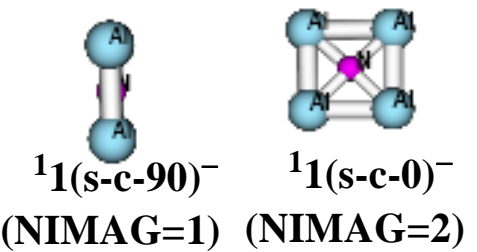

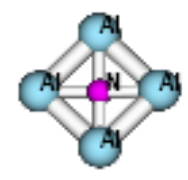

bi

6.50

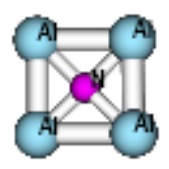

ti

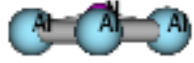

$\mathbf{1}_{1}(\mathbf{f}-\mathrm{s}-90)^{-}$

7.96

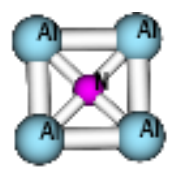

ఉ)

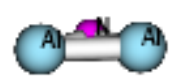

$\mathbf{1}_{\mathbf{1}(\mathbf{f}-\mathrm{s}-\mathbf{0})^{-}}$

8.05
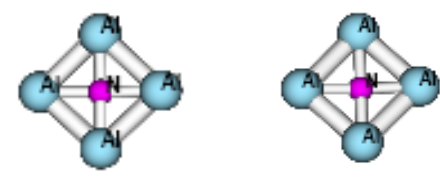

Gi
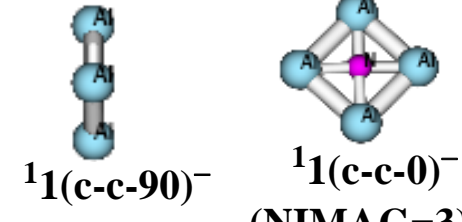
$(\mathrm{NIMAG}=2) \quad(\mathrm{NIMAG}=3)$

12.01

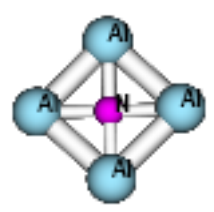

(4)

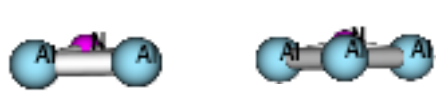

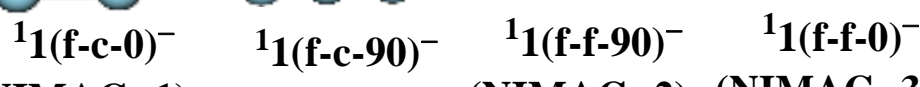
$(\mathrm{NIMAG}=1) \quad(\mathrm{NIMAG}=2) \quad(\mathrm{NIMAG}=2) \quad(\mathrm{NIMAG}=3)$

14.72

14.74

18.56

18.74

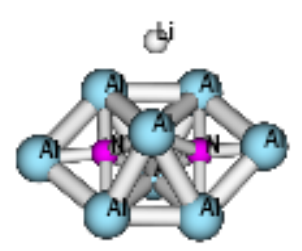

$-3.97$

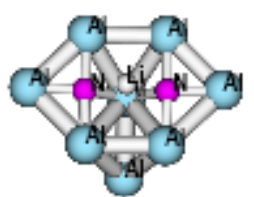

$-0.87$

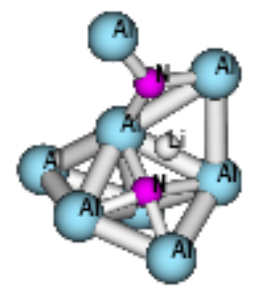

$-3.43$

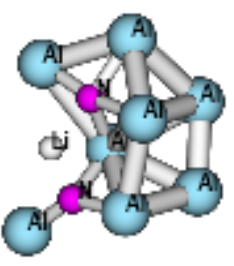

$-3.06$

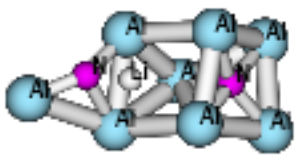

$-2.33$

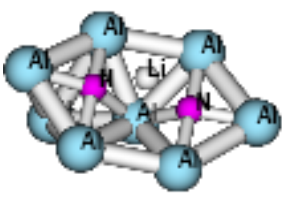

$-1.37$

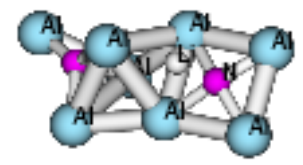

0.57

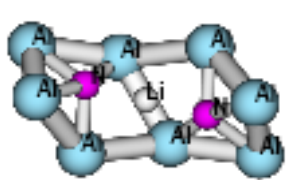

19.79

a) ${ }^{1}\left[\left(\mathrm{Al}_{4} \mathrm{~N}\right)_{2} \mathbf{L i}\right]^{-}$ 
3. The calculated properties of $\mathrm{N}_{2} \mathrm{Al}_{8} \mathrm{Na}^{-}$

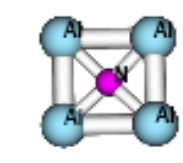

Ta

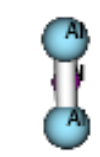

${ }^{1} \mathbf{1}_{(\mathrm{s}-\mathrm{s}-90)^{-}} \quad(\mathrm{NIMAG}=\mathbf{1})$

0.00

0.90

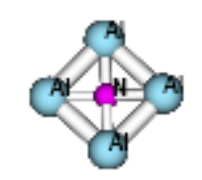

№

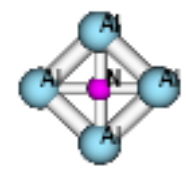

Cia

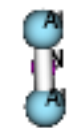

${ }^{1} 1(\mathrm{~s}-\mathrm{c}-90)^{-}{ }^{1} 1$ (s-c-0) $^{-}$ $(\mathrm{NIMAG}=1)(\mathrm{NIMAG}=\mathbf{2}$

4.98

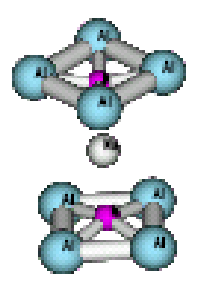

${ }^{1} \mathbf{1}_{(\mathbf{f}-\mathbf{f}-90)^{-}}$

21.92
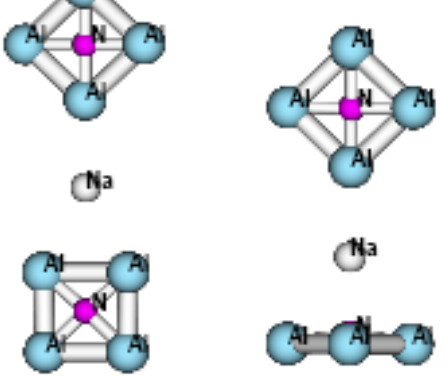

1

1 (f-c-90) ${ }^{-}$
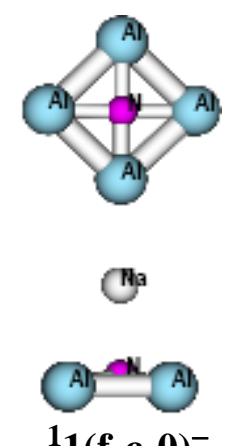

${ }^{1} \mathbf{1}(\mathbf{f}-\mathrm{c}-\mathbf{0})^{-}$ $(\mathrm{NIMAG}=1)$

15.89

15.91

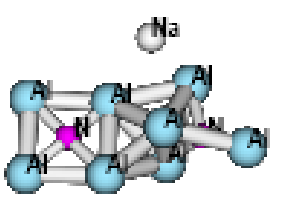

$-1.43$

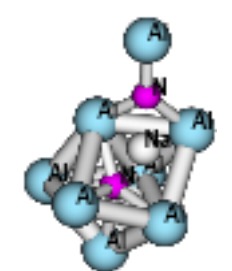

0.89
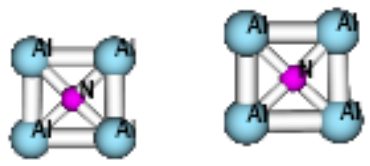

ㄱa
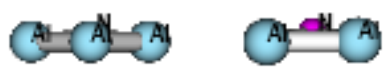

$\left.{ }^{1} \mathbf{1}^{(f-s-90)}\right)^{-}{ }^{1} \mathbf{1}$ (f-s- $\left.^{-0}\right)^{-}$

9.98

10.03
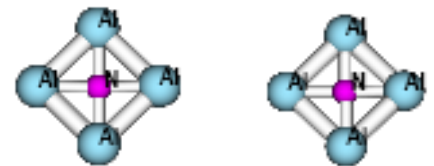

Cy

N3a

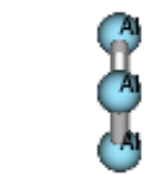

${ }^{1} 1(\mathrm{c}-\mathrm{C}-90)^{-} \quad{ }^{1} \mathbf{1}(\mathrm{c}-\mathrm{c}-0)^{-}$

(NIMAG=2) (NIMAG=3)

10.73

11.02

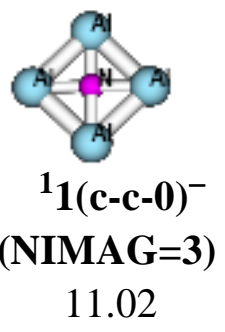

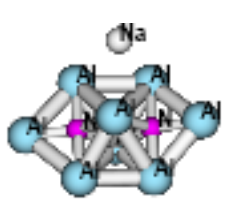

$-5.30$

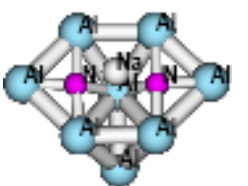

13.80

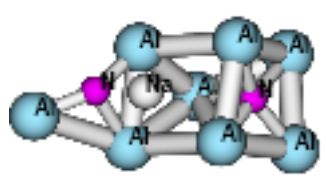

2.63

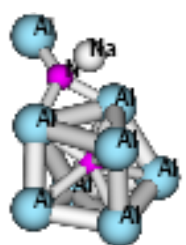

3.30

b) ${ }^{1}\left[\left(\mathrm{Al}_{4} \mathrm{~N}\right)_{2} \mathrm{Na}\right]^{-}$

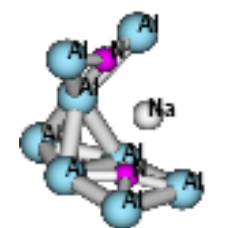

17.59 
4. The calculated properties of $\mathrm{N}_{2} \mathrm{Al}_{8} \mathrm{~K}^{-}$
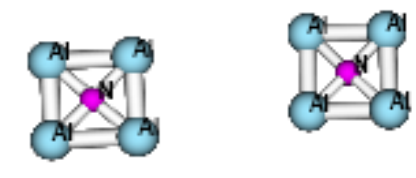

0

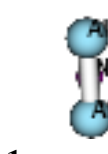

1)(s-s-90)

0.00

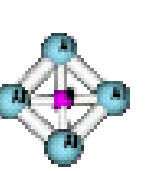

3

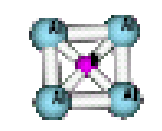

${ }^{1} 1(\mathrm{~s}-\mathrm{c}-\mathbf{0})^{-}$ (NIMAG =2) (NIMAG=1)

4.39

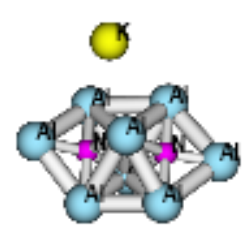

$-4.54$
O

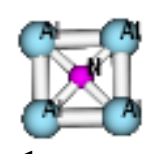

$$
{ }^{1} \text { (s-s-0) }^{-}
$$

(NIMAG $=1$

0.43

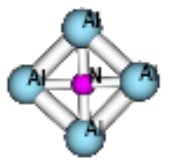

3

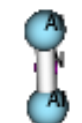

(NIMAG=1)

4.15

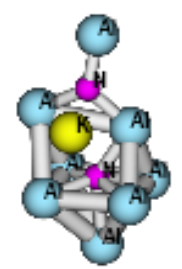

0.65

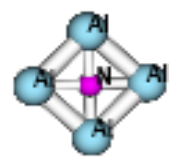

O

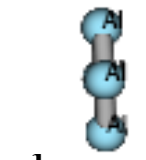

${ }^{1} 1(\mathrm{C}-\mathrm{C}-90)^{-}$

(NIMAG-3) (NI(c-c-0)

8.79

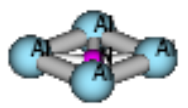

0

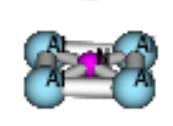

${ }^{1} 1(f-f-90)^{-}$ $(\mathrm{NIMAG}=3) \quad(\mathrm{NIMAG}=5)$

17.12

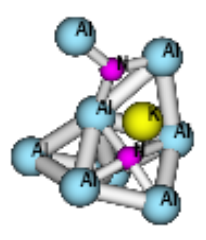

2.80

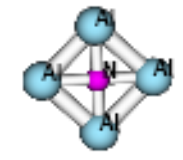

0

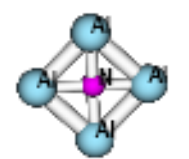

${ }^{1} 1(\mathrm{c}-\mathrm{c}-0)^{-}$

8.95
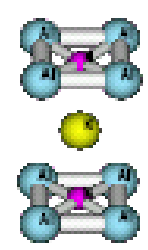

\subsection{2}

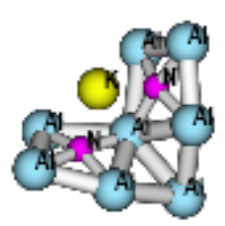

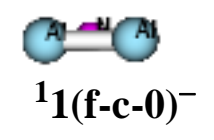

(NIMAG=2) (NIMAG=4)

12.55
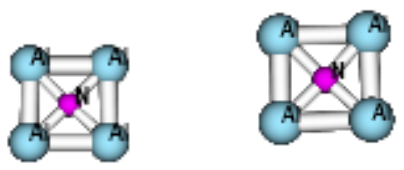

0

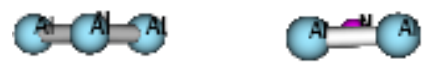

${ }^{1} \mathbf{1}_{(\mathrm{f}-\mathrm{s}-90)}{ }^{-} \quad{ }^{1} \mathbf{1}(\mathrm{f}-\mathrm{s}-\mathbf{0})^{-}$ (NIMAG=1) (NIMAG=1)

8.03

8.00

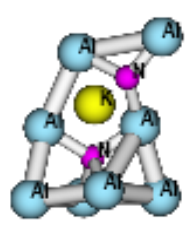

13.58

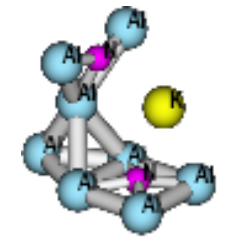

15.53

c) ${ }^{1}\left[\left(\mathrm{Al}_{4} \mathrm{~N}\right)_{2} \mathrm{~K}\right]^{-}$ 

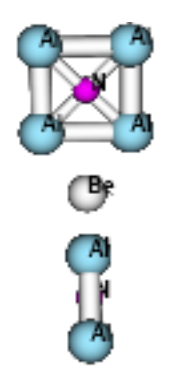

${ }^{1} \mathbf{1}(\mathrm{s}-\mathrm{s}-90)$ 0.00

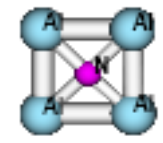

Be

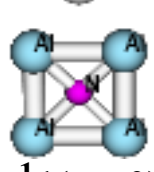

${ }^{1} 1(\mathrm{~s}-\mathrm{s}-0) \quad{ }^{1}$ 1(c-c-90)

(NIMAG=1) (NIMAG=2)

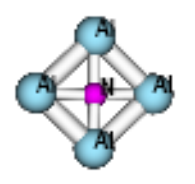

Be

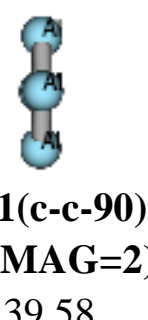

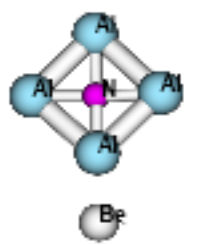

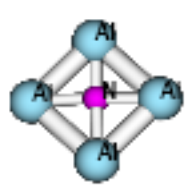

1 1(c-c-0)

$(\mathrm{NIMAG}=3)(\mathrm{NIMAG}=1)(\mathrm{NIMAG}=2)$

43.43
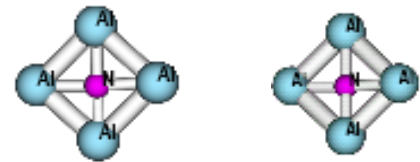

(B)

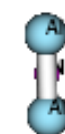

(A)

(s-c-90)

18.60

24.36

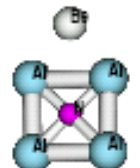

1 1(s-c-0)
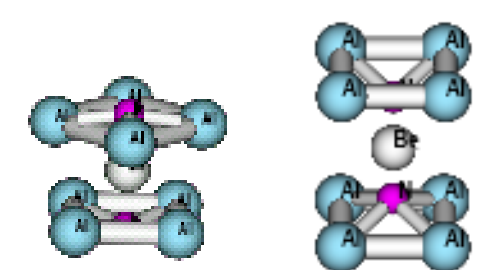

(B)
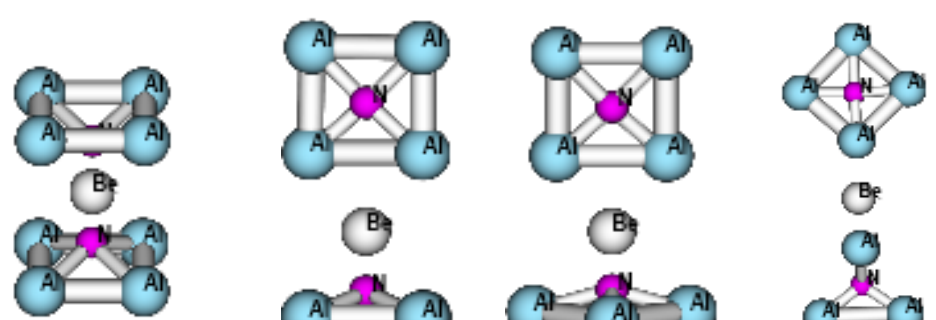

(B)

(B)

(B)
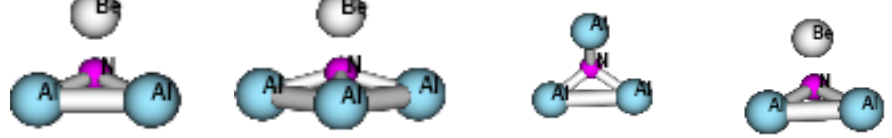

${ }_{1}^{1}$ (f-f-90)

${ }^{1} \mathbf{1}(f-f-0)$

$$
1 \text { 1(f-s-0) }
$$

${ }^{1} \mathbf{1}(\mathrm{f}-\mathrm{s}-\mathbf{9 0})$

${ }_{1}^{1}$ (f-c-90)

${ }^{1} \mathbf{1}(f-c-0)$ $(\mathrm{NIMAG}=10) \quad(\mathrm{NIMAG}=5) \quad(\mathrm{NIMAG}=3)$ 60.86

24.82

10.92

10.23

37.13

31.07

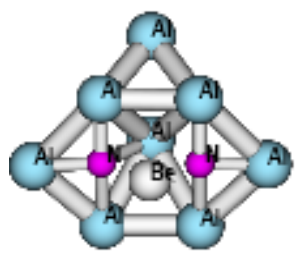

$-11.65$

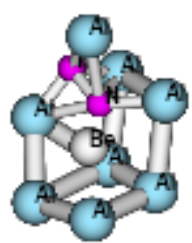

61.33

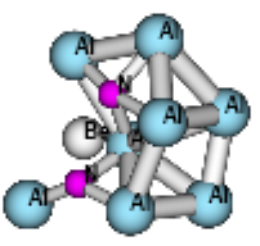

$-10.23$

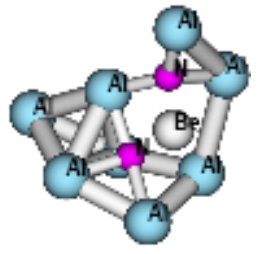

$-7.55$

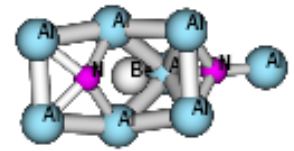

$-4.47$

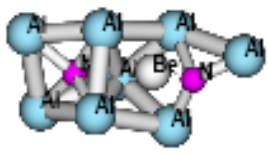

$-4.03$

d) ${ }^{1}\left[\left(\mathrm{Al}_{4} \mathrm{~N}\right)_{2} \mathrm{Be}\right]$ 

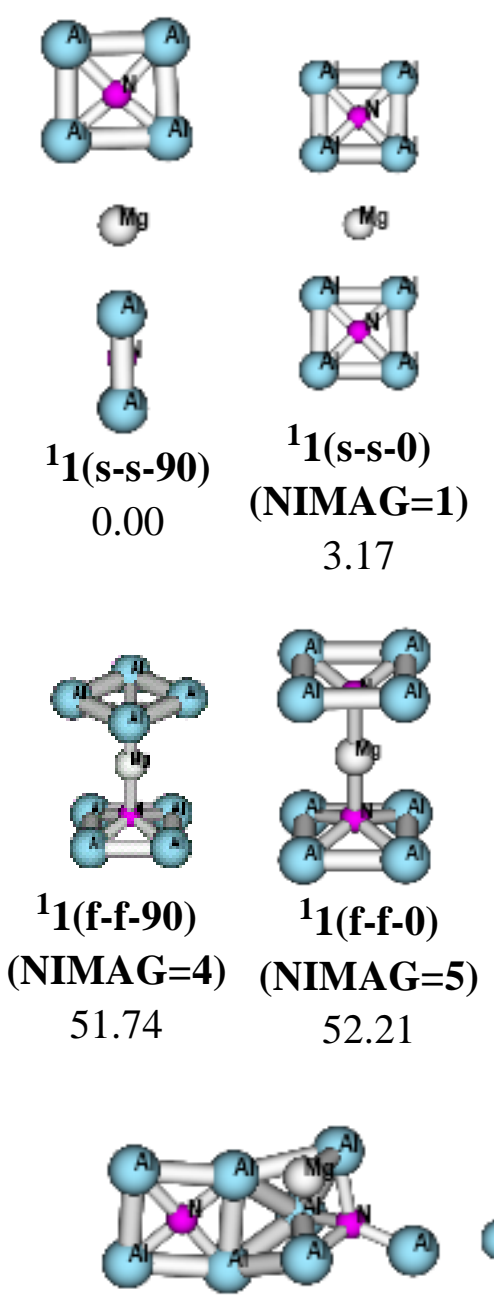

$-2.11$
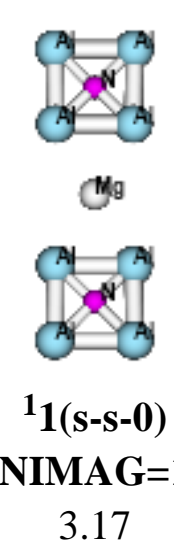

$M A G=5)$

52.21

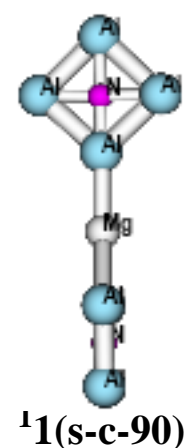

(NIMAG=1)
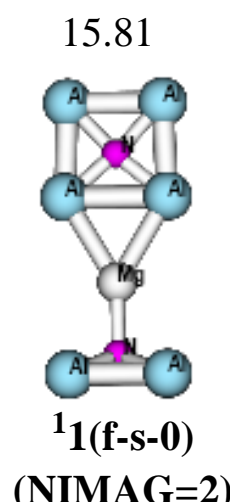

22.71

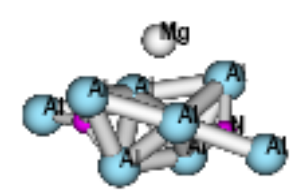

5.79
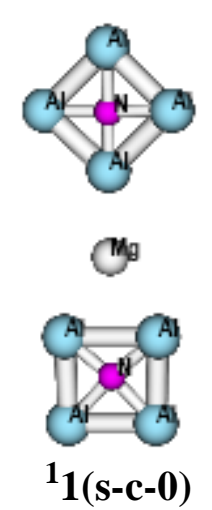

(NIMAG=2)

17.53

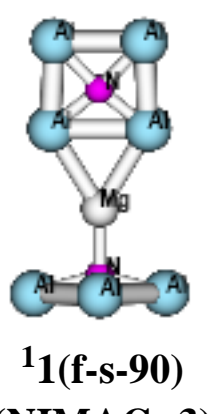

(NIMAG=3)

22.76

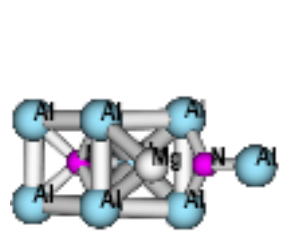

8.71

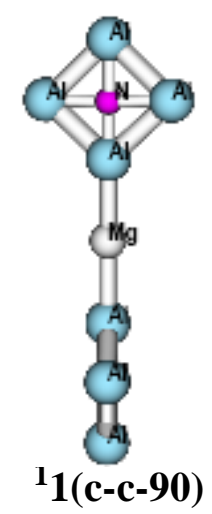

$(\mathrm{NIMAG}=2) \quad(\mathrm{NIMAG}=3)$
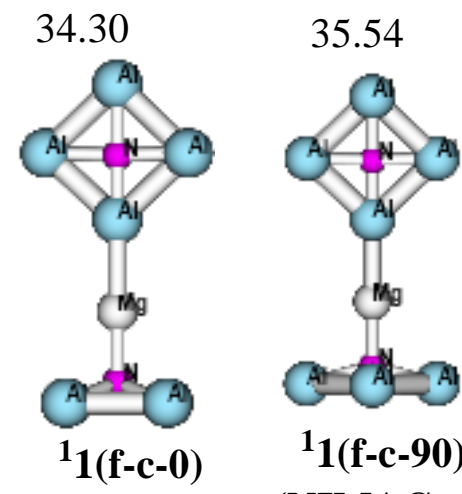

$1 \mathbf{1}(\mathrm{f}-\mathrm{c}-90)$

(NIMAG=3) (NIMAG=4)

42.83

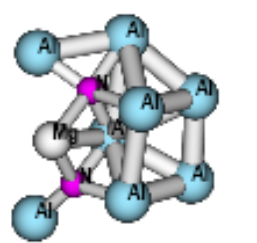

11.06

e) ${ }^{1}\left[\left(\mathrm{Al}_{4} \mathrm{~N}\right)_{2} \mathrm{Mg}\right]$ 

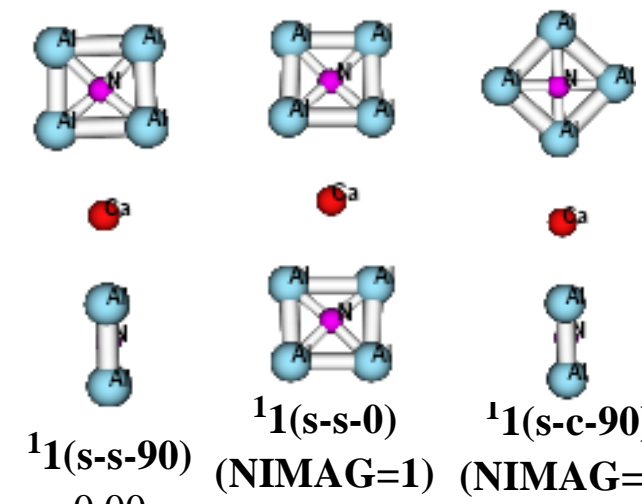

${ }_{1}^{1}$ (s-s-0)

0.00

(NIMAG=1)

1.07

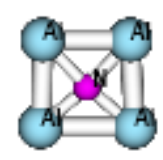

Oa

0
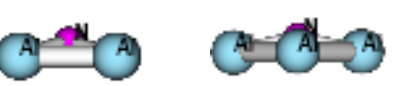

$$
{ }^{1} 1 \text { (f-s-0) }{ }^{1} 1 \text { (f-s-90) }
$$

(NIMAG=2) (NIMAG=3) 16.97

17.08

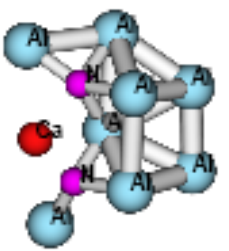

$-9.16$

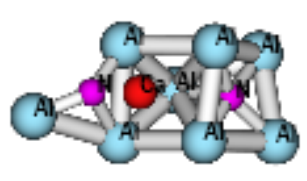

$-2.28$

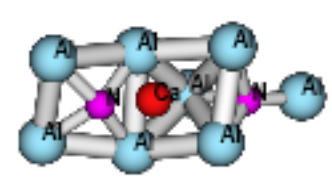

$-1.50$
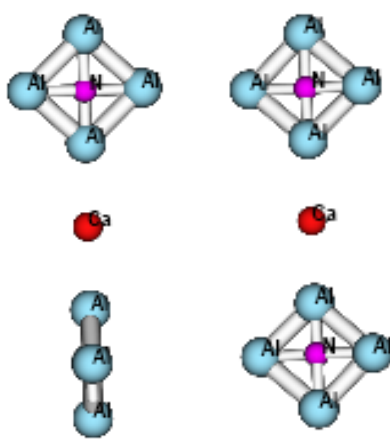

${ }_{1}^{1}$ (c-c-90) ${ }^{1} 1$ (c-c-0)

${ }^{1} 1(\mathrm{~s}-\mathrm{C}-90) \quad{ }^{1} 1(\mathrm{~s}-\mathrm{c}-0)$

14.47

29.49

29.85
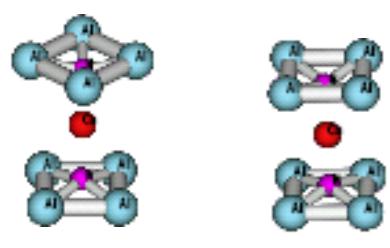

${ }^{1} \mathbf{1}(f-f-90) \quad{ }^{1} 1(f-f-0)$

$(\mathrm{NIMAG}=4)(\mathrm{NIMAG}=5) \quad(\mathrm{NIMAG}=6) \quad(\mathrm{NIMAG}=7)$

34.59

35.58

f) ${ }^{1}\left[\left(\mathrm{Al}_{4} \mathrm{~N}\right)_{2} \mathrm{Ca}\right]$ 
8. The calculated properties of ${ }^{1} \mathrm{NAl}_{4} \mathrm{LiCp}{ }^{-}$

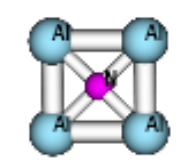

(4)

H.CBg $-C_{-H}^{-H}$

${ }^{1} \mathbf{1}(\mathbf{f}-\mathrm{s}-1)^{-}$

0.00

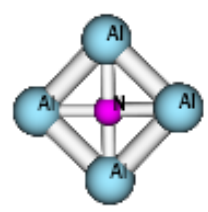

(c)

$$
\begin{aligned}
& { }^{1} \mathbf{1}(\mathbf{f}-\mathrm{s}-\mathbf{1})^{-} \\
& 2.50 \\
& \text { NIMAG=1 }
\end{aligned}
$$
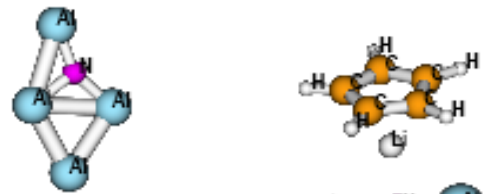

bi

Hatecter

17.78

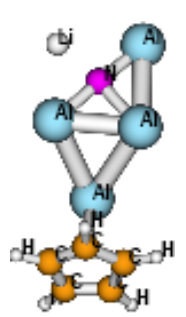

39.72

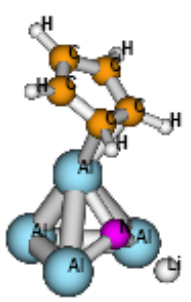

45.06

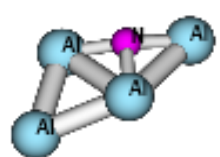

17.82

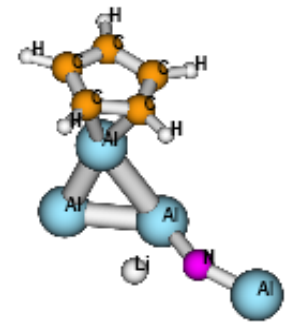

40.96

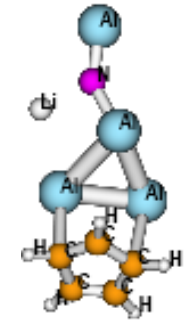

47.68

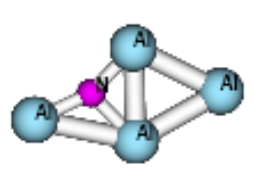

(4)

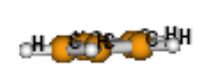

19.94

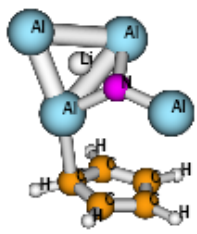

41.31

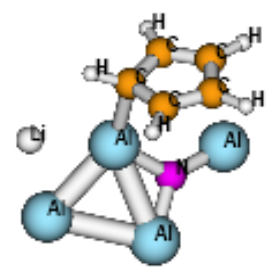

47.94
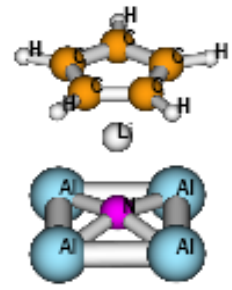

${ }^{1} \mathbf{1}(\mathbf{f}-\mathbf{f})^{-}$

2.84

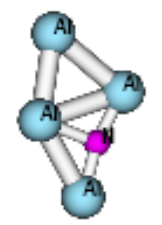

ti

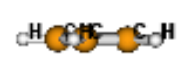

21.36

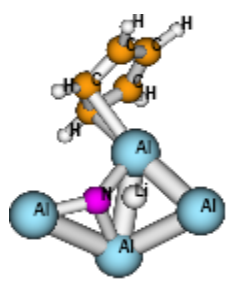

41.94

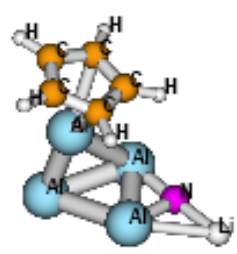

52.81

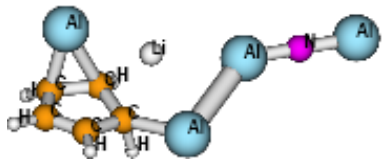

84.10

a) ${ }^{1}\left[\mathrm{CpLi}\left(\mathrm{Al}_{4} \mathrm{~N}\right)\right]^{-}$ 
9. The calculated properties of ${ }^{1} \mathrm{NAl}_{4} \mathrm{NaCp}^{-}$

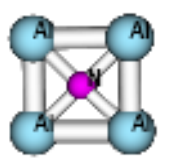

Cia

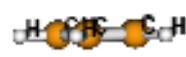

${ }^{1} \mathbf{1}(\mathbf{f}-\mathrm{s}-\mathbf{1})^{-}$

0.00

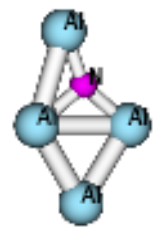

(1)

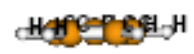

18.80

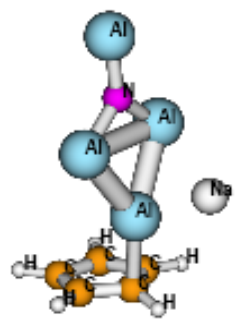

27.50

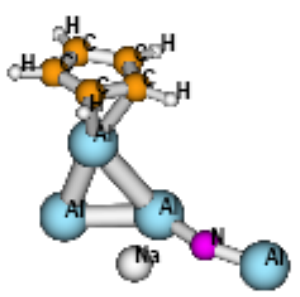

37.46

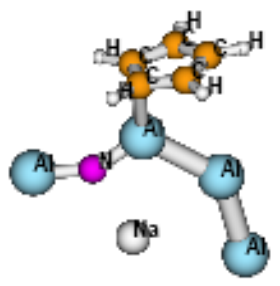

51.44

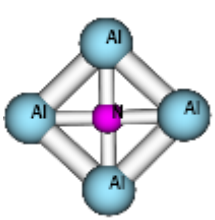

(N.)

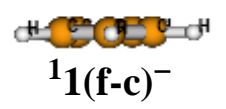

3.43

NIMAG=1

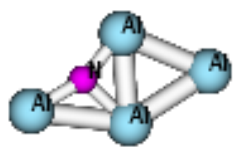

Ca

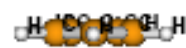

20.40

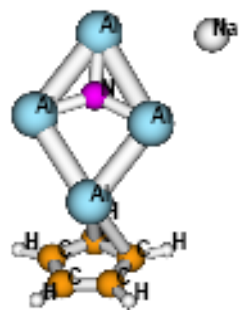

28.06

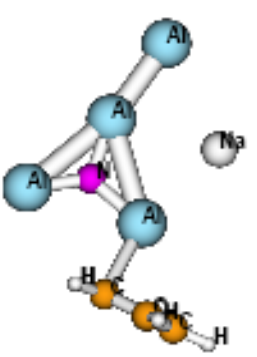

38.86

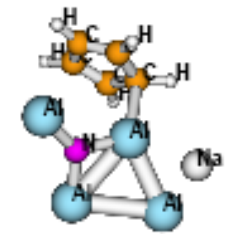

32.73

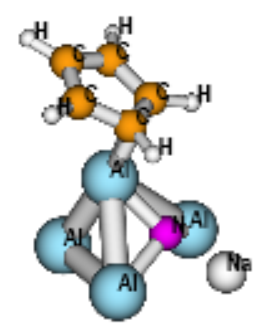

41.74

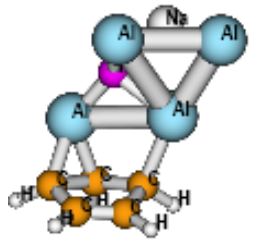

82.27

(A)

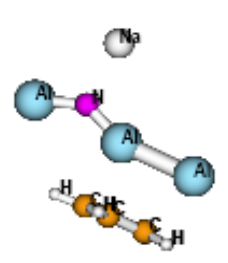

71.91

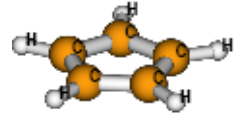

(N3)

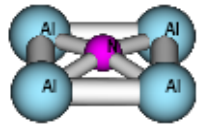

${ }^{1} \mathbf{1}(\mathbf{f}-\mathbf{f})^{-}$

7.86

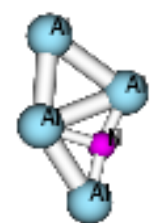

C49

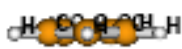

22.96

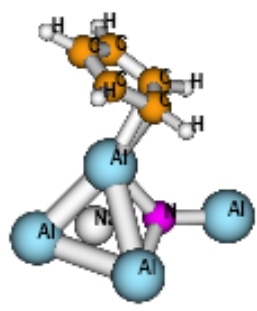

35.65

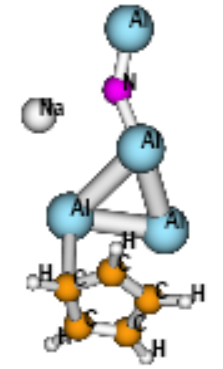

44.92

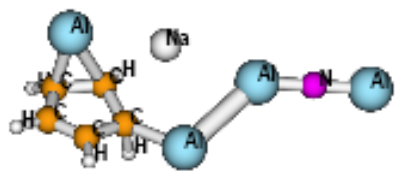

85.57

b) ${ }^{1}\left[\mathrm{CpNa}\left(\mathrm{Al}_{4} \mathrm{~N}\right)\right]^{-}$ 
10. The calculated properties of ${ }^{1} \mathrm{NAl}_{4} \mathrm{KCp}^{-}$

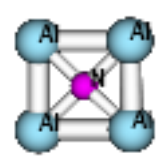

O

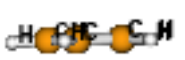

${ }^{1} \mathbf{1}(\mathrm{f}-\mathrm{s})^{-}$

0.00

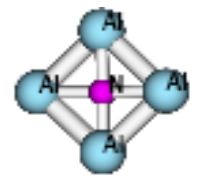

0

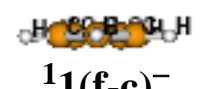

${ }^{1} \mathbf{1}(\mathbf{f}-\mathrm{c})^{-}$

2.81

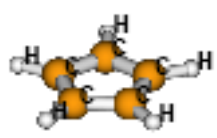

(1)

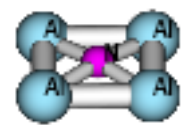

$\mathbf{1}_{\mathbf{1}(\mathbf{f}-\mathbf{f})^{-}}$

6.36

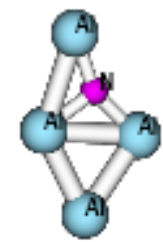

NIMAG $=1$

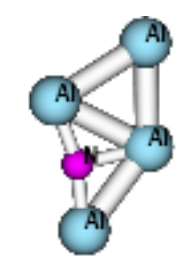

G

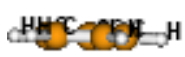

20.46

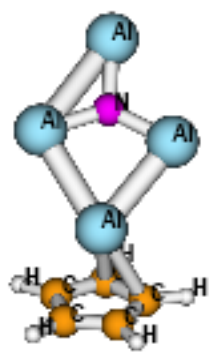

32.97
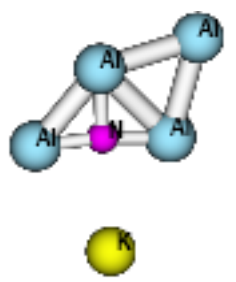

(3)

HCE CAS - $^{H}$

22.06

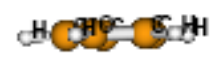

23.25

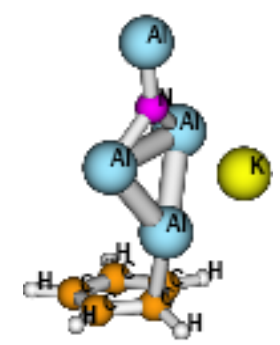

31.47

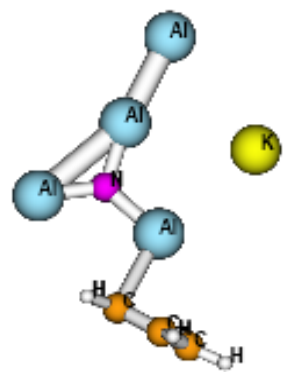

43.64

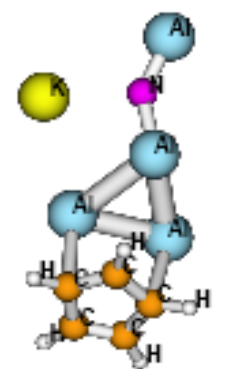

48.04

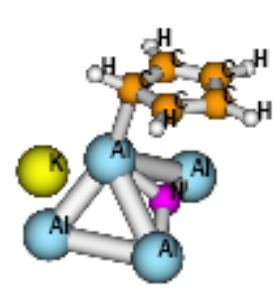

37.09

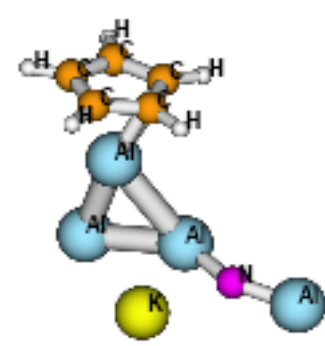

39.63

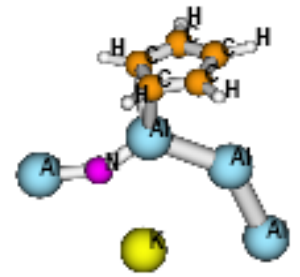

55.74

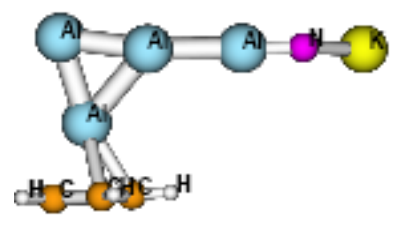

102.92

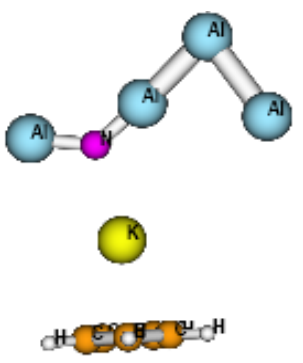

49.62

c) ${ }^{1}\left[\mathrm{CpK}\left(\mathrm{Al}_{4} \mathrm{~N}\right)\right]^{-}$ 
11. The calculated properties of ${ }^{1} \mathrm{NAl}_{4} \mathrm{BeCp}$

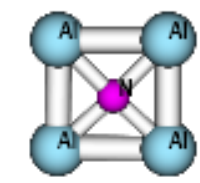

(B)

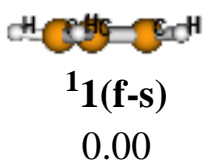

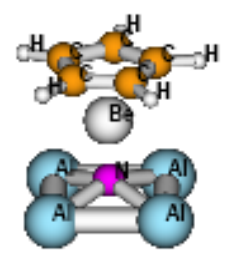

${ }^{1} \mathbf{1}(\mathbf{f}-\mathbf{f})$

2.62

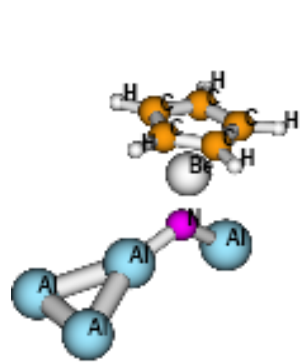

9.76

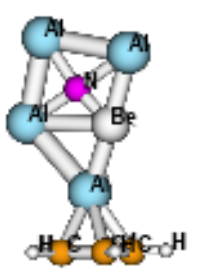

16.73

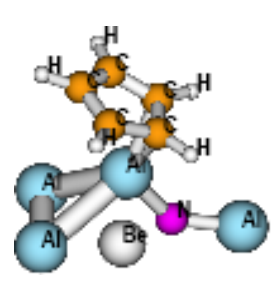

27.05
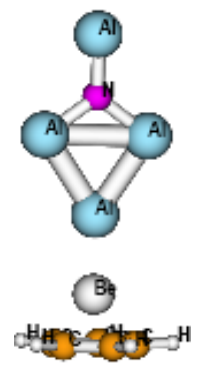

10.43
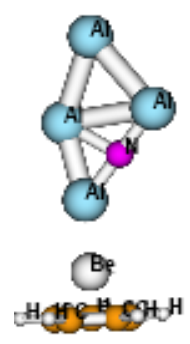

21.24

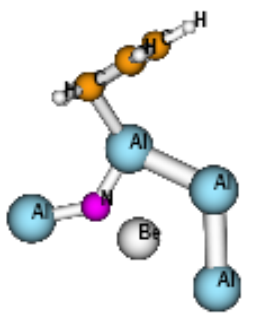

33.00
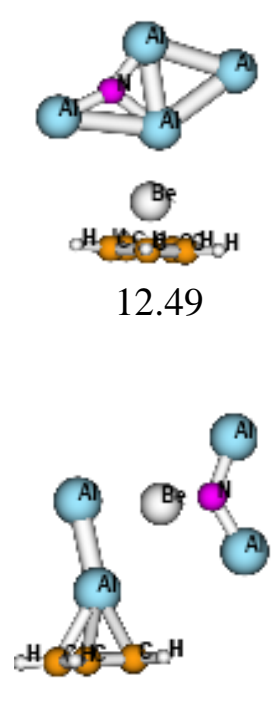

22.12

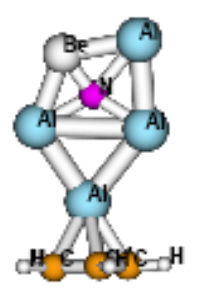

35.15

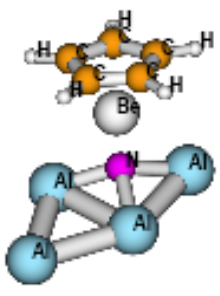

12.83

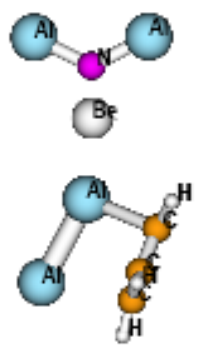

26.82

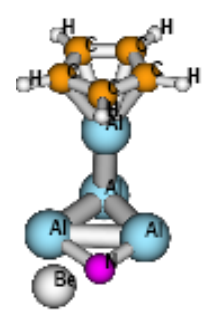

40.78

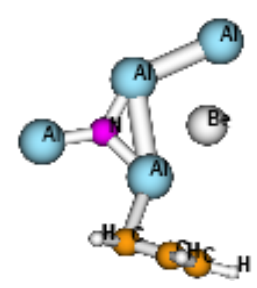

49.77

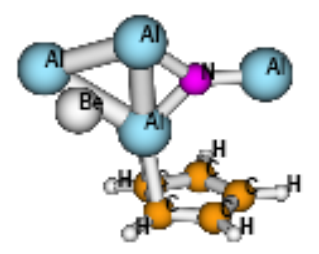

52.51

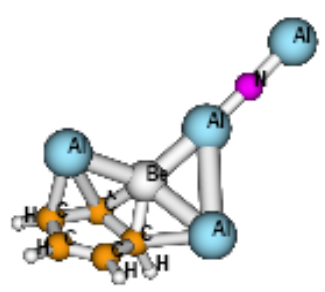

81.07

d) ${ }^{1}\left[\mathrm{CpBe}\left(\mathrm{Al}_{4} \mathrm{~N}\right)\right]$ 
12. The calculated properties of ${ }^{1} \mathrm{NAl}_{4} \mathrm{MgCp}$
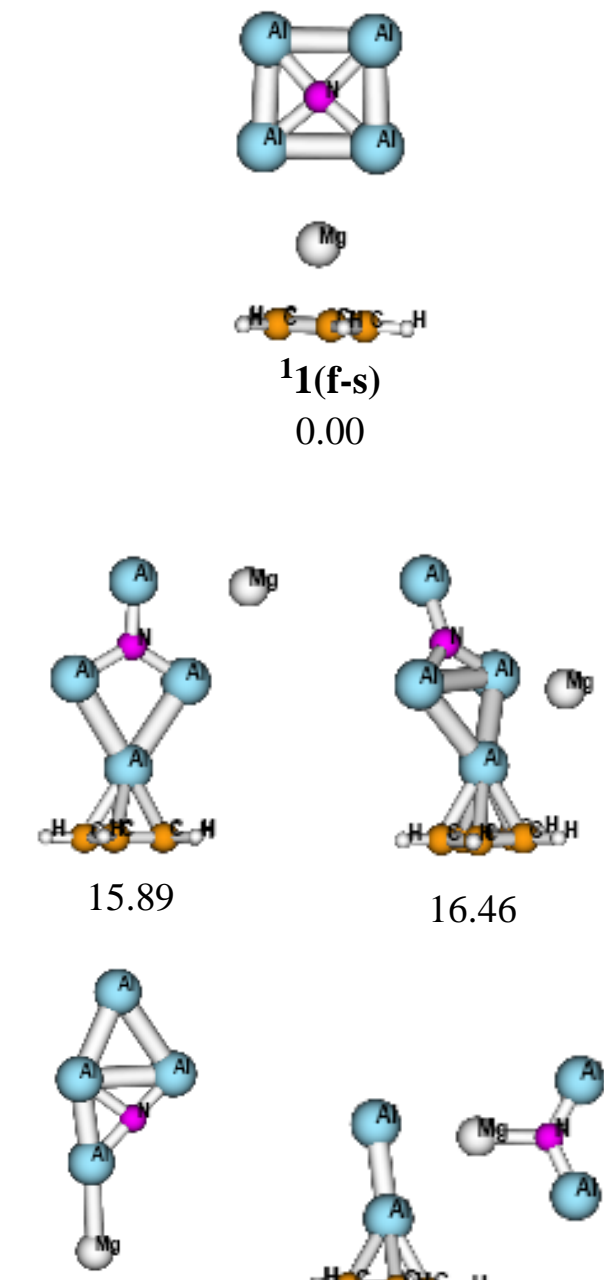

Hereeght

28.22

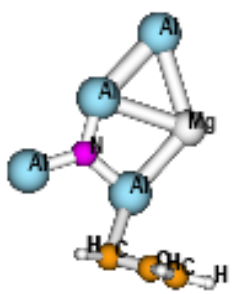

35.40

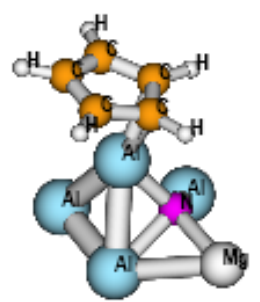

44.61

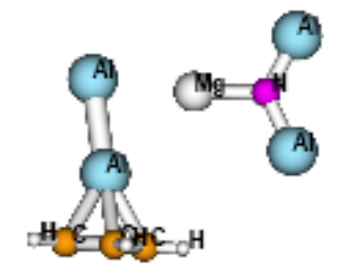

29.23

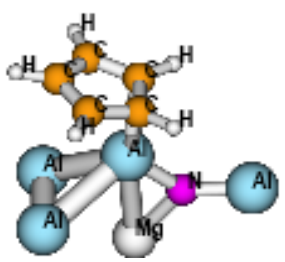

36.97

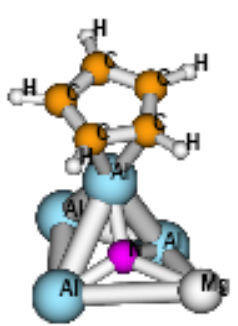

44.61

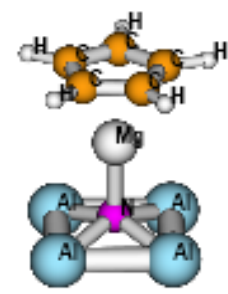

$\mathbf{1}_{1}(\mathbf{f}-\mathbf{f})$

20.19
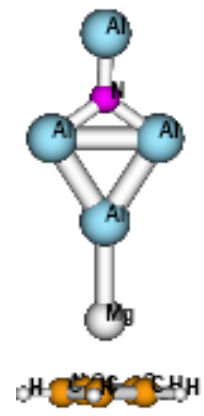

16.63

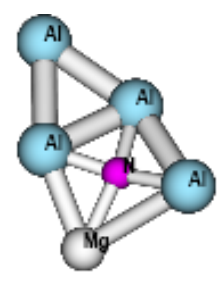

H.

25.32

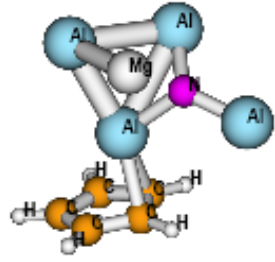

33.54

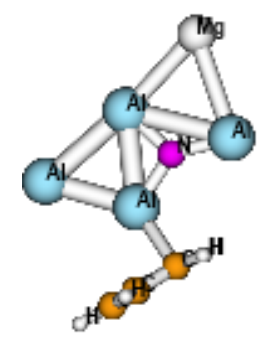

34.42

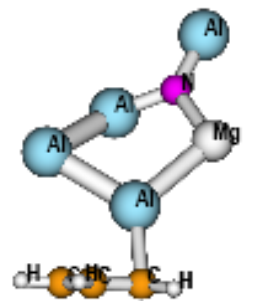

37.24

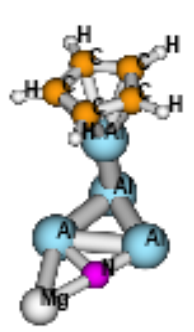

49.14

e) ${ }^{1}\left[\mathrm{CpMg}\left(\mathrm{Al}_{4} \mathrm{~N}\right)\right]$ 
13. The calculated properties of ${ }^{1} \mathrm{NAl}_{4} \mathrm{CaCp}$
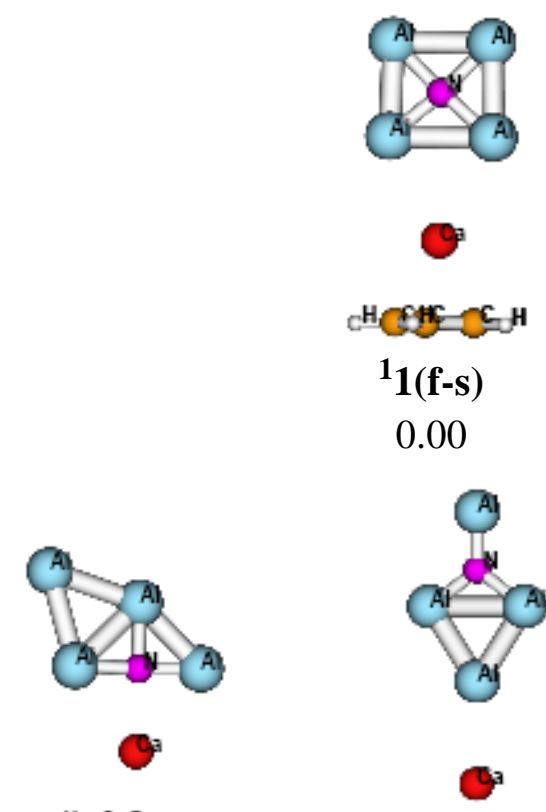

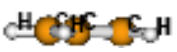

16.81

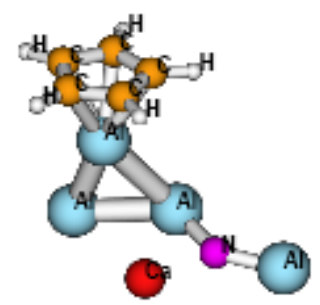

35.87

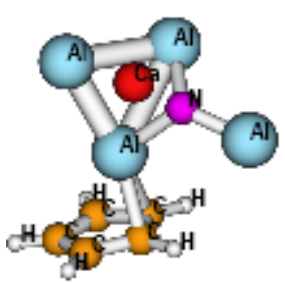

43.62
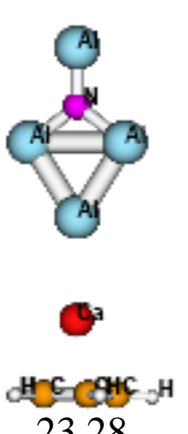

23.28

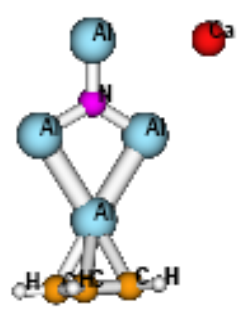

39.43

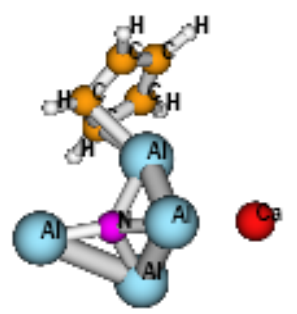

47.81

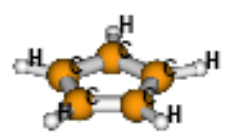

0

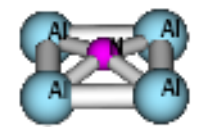

${ }^{1} \mathbf{1}(\mathbf{f}-\mathbf{f})$

15.38
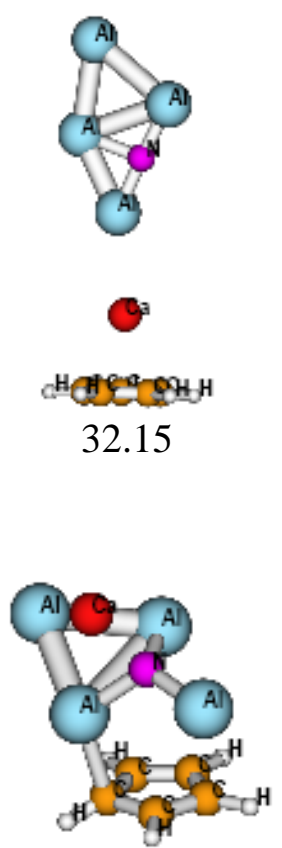

42.53

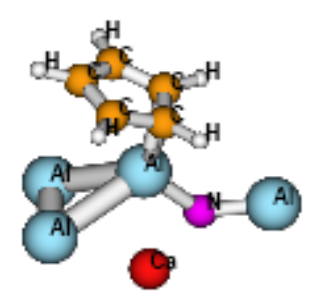

43.26

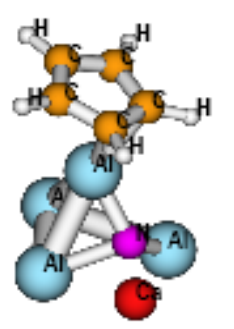

52.43

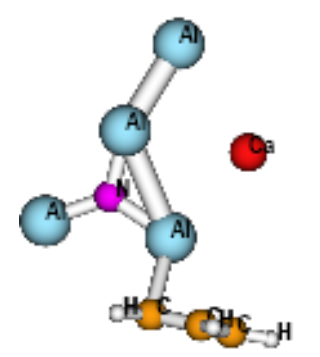

53.79

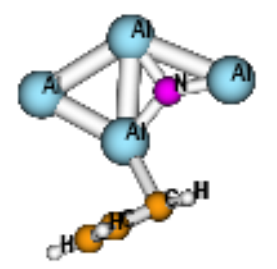

56.20

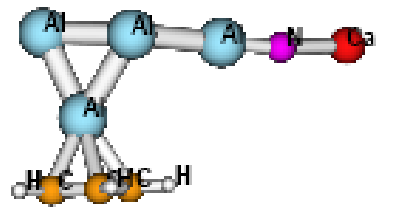

100.46

f) ${ }^{1}\left[\mathrm{CpCa}\left(\mathrm{Al}_{4} \mathrm{~N}\right)\right]$ 
14. The calculated properties of various extend system

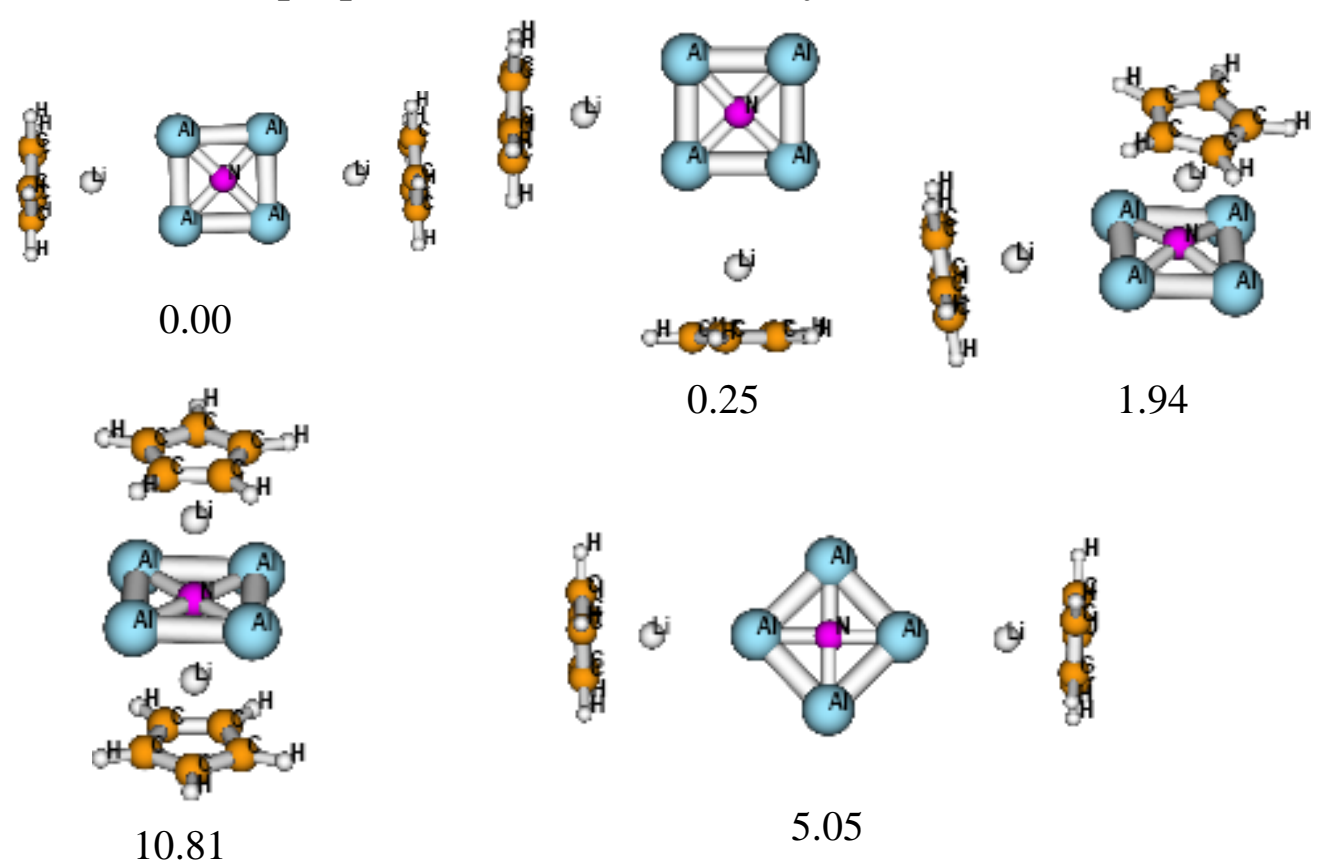

a) $\left[\mathrm{Cp}_{2} \mathrm{Li}_{2}\left(\mathrm{Al}_{4} \mathrm{~N}\right)\right]^{-}$

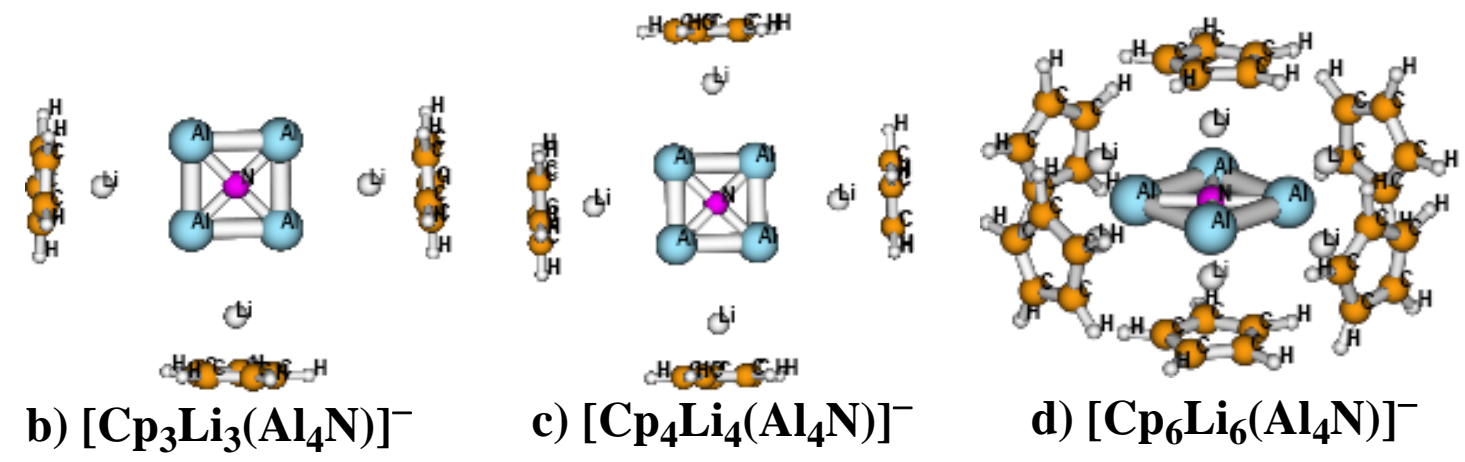




$$
\text { (i) }
$$

